\title{
PHYSICS OF THE SOLID STATE
}

$\mathrm{T}$ HE 1957 spring Meeting of the Physical Society was held at the University of Nottingham during April 8-10, the general topic being "The Physics of the Solid State". About one hundred and fifty members and guests were present. Some twenty or so papers were given, the majority falling into one or other of the two broad categories of magnetism and semiconduction.

The meeting opened with a welcome and talk on Bitter figures by Prof. L. F. Bates (University of Nottingham), who gave a rapid, broad and wellillustrated survey of the different types of patterns which can be observed using this technique. While the underlying ideas are fairly simple, the detailed understanding of the reasons why such beautiful patterns occur is a problem of considerable magnitude. Good progress is, however, being made, and the recent development of methods for observing the patterns using the electron microscope should greatly assist. This was followed by an account of the application of neutron diffraction to magnetic problems, by G. E. Bacon (Atomic Energy Research Establishment, Harwell), in which a brief survey of the technique was followed by a discussion of some of the current and projected applications. This use of neutrons has been invaluable in helping to solve magnetic problems, and it was therefore of the greatest interest to hear about the difficulties which have to be overcome, for it is all too easy to assume that any magnetic problem can be solved by this method. In fact, the magnetic scattering is often quite small compared with the scattering from various other sources, and a good deal of very careful analysis has to be performed. It was pointed out that the study of inelastic scattering could be of particular value in deciding between the various theories of magnetism and that preliminary results were being obtained for iron. After some discussion the formal proceedings of the day ended, though the informal discussions, which form such a valuable part of these meetings, continued over sherry and dinner.

The second day's session was devoted to the magnetism of metals and alloys, a subject which was introduced by K. W. H. Stevens (University of Nottingham), who used the rare-earth metals to illustrate why there is at present so much interest in the part played by conduction electrons in magnetism. J. Owen (University of Oxford) then gave an account of his investigation, at Berkeley, of copper-rich copper-manganese alloys, using susceptibility, and electronic and nuclear resonance measurements. While the results are not yet fully understood, it appears that the manganose atoms possess large localized moments, that antiferromagnetism sets in at low temperatures, and that there is a polarization of the conduction electrons which decreases rapidly with distance from the manganese atoms. The study of this particular system has proved so rewarding that it is to be hoped that further alloys will be investigated along these lines. Although F. E. Hoare (University of Leeds) had not used the same methods, he had found anomalies in the variations with temperature of the magnetic susceptibilities and electronic specific heats, in the families of alloys of palladium with rhodium and silver, and platinum with iridium and gold. It is not clear how these can be interpreted on the band theory of metals, unless there is an exchange interaction dependent upon temperature. There was then a change to more macroscopic properties of ferromagnetic alloys, Prof. W. Sucksmith (University of Sheffield) giving an account of the remarkable properties of precipitates of fairly large ferromagnetic particles in non-magnetic matrices, with particular reference to iron-copper, nickel-gold and cobalt-copper systems. The interpretation of the data is far from easy, depending on a number of uncertain parameters, but E. P. Wohlfarth (Imperial College of Science and Technology) showed that it is possible to make substantial progress.

The next session was concerned with non-metallic magnetic systems, J. S. Smart (United States Office of Naval Research, London) reviewing Neel's theory of ferrimagnetism and recent experimental work which has so beautifully confirmed it. He directed attention to some of the difficulties about the nonvanishing of $\delta M / \delta T$ at absolute zero in some substances, and pointed out that although it had been postulated that there may be angles between spins on the same sub-lattice, this has not yet been confirmed by experiment. K. J. Standley (University of Nottingham) then gave a talk about his measurements of the dispersion of the dielectric constant and the apparent resistivity in certain ferrites. These have been made over a wide range of frequencies and at a number of different temperatures and show unexpected behaviour, which may partly be associated with the purely practical difficulty of measuring the dielectric constant when the permeability is large, but which may also indicate the existence of permanent electric dipole moments. W. Marshall (Atomic Energy Research Establishment, Harwell) gave a theoretical interpretation, using two exchange interactions of opposite signs, the dominant one being antiferromagnetic, of the observation on hydrated cupric chloride that the maximum susceptibility occurs at a temperature which is definitely above the Néel temperature. It seems that appreciable shortrange order exists above this temperature, a conclusion which is consistent with the shape of the specific heat anomaly. P. L. Smith reported the results of specific heat measurements at helium temperatures on cerium ethyl sulphate and a number of chloroiridates, all of which have been studied in some detail by electronic resonance. It seems that even where a Schottky type of anomaly is expected the shape is not always right, nor is the lattice specific heat always proportional to $T^{\mathbf{8}}$.

On the evening of April 9 the Guthrie Lecture was delivered by Prof. H. C. Urey (University of Chicago), on "Some Chemical Limitations on Theories of the Solar System". 'This Lecture is given annually by a distinguished scientist in memory of the founder of the Physical Society, an innovation this year being to hold it in the provinces in the course of a conference. Among those who heard the Lecture was Miss Guthrie, the founder's daughter. Prof. Urey treated his audience to a most stimulating and wideranging account of some of the problems which he has met in trying to interpret our knowledge about such topics as the relative abundances of the elements, the compositions of the meteorites and the theories 
of the origins of the solar system, in terms of ordinary physical and chemical ideas. It was typical of Prof. Urey that although his talk was not primarily about magnetism, he nevertheless provided the experts with some rather awkward problems to think about. In the evening an informal conference dinner was held in the dining room of the recently opened Portland building.

The last day of the meeting was devoted mainly to topics related to semiconduction, with the morning's lectures largely concerned with the applications of resonance methods. By way of introduction to this, Prof. M. H. L. Pryce (University of Bristol) gave an elementary and comprehensive survey of the three techniques of cyclotron, paramagnetic and nuclear resonance. Then followed E. H. Rhoderick (Services Electronics Research Laboratory, Baldock), who gave a detailed account of a number of experiments in which nuclear magnetic resonance had been used to study some of the III-V semiconductors. In this the nuclei are used essentially as probes to examine what the electrons are doing, which means that the value of the information depends critically on the electron-nuclear coupling. The III-V compounds are a much more promising field for this type of work than are germanium and silicon, but even so it must seem at times as if Nature is being deliberately unco-operative. Cyclotron resonance was represented by D. M. S. Bagguley (University of Oxford), who reported the observation of signals at $90^{\circ}$ and $65^{\circ} \mathrm{K}$. from two types of carriers in gold-doped germanium. The masses and relaxation times were found to be independent of temperature. Due to time-table changes, the final paper in this family was given later in the day, when E. E. Schneider (King's College, Newcastle) described the application of electronic resonance and optical work to the study of alkali halide crystals. There seems to be evidence of microstructures in these, and by studying the resonances from very small amounts of divalent manganese impurities, a lot of detailed information can be obtained. For example, one of the manganese resonances is so narrow that it can only be concluded that it comes from ions precipitated on grain boundaries. In addition, we heard from G. G. Macfarlane (Royal Radar Establishment, Malvern) and R. J. Elliott (University of Reading) of some recent work which they have been doing on the interpretation of the fine structure in the intrinsic absorption edge in germanium. It appears that a number of mechanisms contribute, and a first step is to break down the observations into the parts due to the various processes. These have then been studied in detail, particularly the part played by excitons, and a very satisfactory picture of the whole phenomenon has resulted. The final contribution of the moming came from A. L. Parsons, who described his recent theories about diamagnetism.

The first part of the afternoon session was given over to the chemists, beginning with Prof. D. $D$. Eley (University of Nottingham) describing his work on organic semiconductors. In these, excited electrons can travel fairly freely through the molecules, but are impeded by having to tunnel from one molecule to the next. As might be expected, the energy gap is correlated with the excitation energy of the molecule. As typical of the systems being studied, we may mention metal-free phthalocyanine, the com. plicated substance known more familiarly to physicists as the g-marker, and some of the proteins. Then C. H. L. Goodman (General Electric Company) pro- pounded a number of empirical rules which he has found useful in predicting new inorganic semiconductors, and described various exotic new semiconductors which he had prepared with their help, usually by substituting an $A$ and a $C$ atom for two $B$ atoms, while retaining the same number of electrons. There seems to be no end to this process of producing new semiconductors, but at present it is an open question whether any of them will prove to be as valuable as the more conventional ones. In the same vein, J. C. Woolley (University of Nottingham) described his experiments on making mixed III-V compounds. The literature about this is confusing, but it now seems to be definitely established that InSb-GaSb and InAs-GaAs can be made. It takes a long time to obtain equilibrium, so that annealing over extended periods has been necessary, although recent developments seem to promise that these long times (of the order of months) can probably be much reduced.

The final session consisted of a number of short communications. J. M. Lock (Royal Radar Establishment, Malvern) described his recent susceptibility and specific heat results on some of the rare earth metals, A. E. Liddiard (Átomic Energy Research Establishment, Harwell) spoke about his work on the thermoelectric power of ionic conductors, J. H. Smith (University of Sheffield) reported on the magneto-resistance of $\mathrm{MnAu}_{2}$, and $\mathrm{S}$. Altmann (University of Oxford) gave a progress report on his application of the cellular method to determine the band structure in hexagonal close-packed metals.

Throughout the sessions and the social gatherings in the evenings there was a good deal of discussion and exchange of ideas, and it seemed to be the general opinion that the meeting served a useful purpose in bringing together so many solid-state physicists. The majority of the papers described work being carried out in Britain, and the overall impression was that a lot of good research is going on, but that, except possibly in some of the very largest laboratories, we are rather slow in exploiting techniques which have been recognized elsewhere as being of great potential value in solid-state physics.

No conference report is being prepared, since it is assumed that the original contributions will be submitted for publication in the usual way.

\section{K. W. H. Stevens}

\section{INSTRUMENTS FOR USE IN OCCUPATIONAL HYGIENE}

$T$

HE British Occupational Hygiene Society held its seventh conference in the Canterbury Hall, University College, London, on April 16 and 17 . Eight papers were read dealing with the nature and use of instruments for guarding the health of indus. trial workers in the performance of their various occupations, which often expose them to potential danger if the materials they handle and their environment are not carefully controlled. Throughout the conference a broad view of the subject was taken, both by the authors and those who entered into discussion with them, and general surveys of the various techniques available, together with operational principles, featured more strongly than technical details, though the latter were by no means lacking. 